\title{
A Generalized Class of Estimators for Estimating Population Mean in the Presence of Non-Response
}

\author{
Iram Saleem ${ }^{1}$, Aamir Sanaullah ${ }^{2}$, and Muhammad Hanif ${ }^{1 \star}$ \\ ${ }^{1}$ Department of Statistics, National College of Business Administration and Economics, Lahore, Pakistan \\ ${ }^{2}$ Department of Statistics, COMSATS Institute of Information and Technology, Lahore, Pakistan.
}

\section{ARTICLE INFO}

\section{Article History}

Received 28 Jun 2017

Accepted 13 Jan 2018

\section{Keywords}

Auxiliary variable

Mean square error

Non-response

Stratified random sampling

2000 Mathematics Subject Classification

22E46, 53C $35,57 \mathrm{~S} 20$

\section{ABSTRACT}

Koyuncu and Kadilar proposed an estimator based on single auxiliary variable with complete response in stratified random sampling. In this paper, we extended Koyuncu and Kadilar's estimator to a more generalized class of estimators using twoauxiliary variables in stratified random sampling for the situation of non-response and further introduced its another improved generalized class of estimators. The mathematical conditions under which proposed class of estimators are efficient as compare to Hansen and Hurwtiz estimator, and ratio estimators modified for stratified sampling have been derived. An empirical study has also been carried out to examine the performance of the suggested estimators.

\section{INTRODUCTION}

Non-response refers to the situation, when an investigator fails to get necessary information from some of the units of the selected sample. The problem of non-response was first analyzed by Hansen and Hurwitz [1]. They developed a classical non-response concept to obtain information from the sub-sample of non-response group. An estimator for the population mean in the presence of non-response was constructed and also derived its variance with the optimum sampling fraction for the non-respondents. It is suitable for the surveys, in which first attempt is made on mail questionnaires and second attempt is selected from the non-respondent persons by personnel interviews. Following Hansen-Hurwitz methodology Cochran [2] proposed ratio type estimator for dealing with non-response. Chaudhary et al. [3], Haq and Shabbir [4] and Sanaullah et al. [5] presented some improved estimators for stratified random sampling under non-response.

In order to improve the efficiency of an estimator, auxiliary information is often used to estimate the unknown population mean of study variable. Cochran [6] discussed classical ratio estimators. Further, Cochran [2], Kadilar and Cingi [7], Shabbir and Gupta [8], Koyuncu and Kadilar [9], Sanaullah et al. [10] and Sanaullah et al. [11] utilized auxiliary information under stratified random sampling scheme.

\section{SAMPLING DESIGN AND PROCEDURE DEALING WITH NON-RESPONSE}

Consider a finite population of size $N$ is stratified into $L$ homogenous strata. Let $N_{h}$ be the size of $h$ th stratum $(h=1,2,3, \ldots, L)$ such that $\sum_{h=1}^{L} N_{h}=N$ and $\left(y_{h i}, x_{h i}, z_{h i}\right)$ be the observations of study variable $y$ and auxiliary variables $x$ and $z$ on the $i$ th unit of $h$ th stratum, respectively. Let $\bar{y}_{h}, \bar{x}_{h}$ and $\bar{z}_{h}$ be the sample means of $h$ th stratum corresponding to the population means $\bar{Y}_{h}, \bar{X}_{h}$ and $\bar{Z}_{h}$ respectively. Usually it is

${ }^{\star}$ Corresponding author. Email: dmianhanif@gmail.com 
not possible to collect complete information from all the units selected in the sample $n_{h}\left(\sum_{h=1}^{L}, n_{h},=, n\right)$. Let $n_{h(1)}$ units from a sample of $n$ h provide their responses and $n_{h(2)}$ units do not. Adapting Hansen and Hurwitz [1] sub-sampling methodology, a sub-sample of size $r_{h}$ $\left(r_{h}=\frac{n_{h(2)}}{f_{h}} ; f h>1\right)$ from $n_{h(2)}$ non-respondents group is selected at random and $1 / f h$ denotes the sampling fraction among the non-respondent group in the $h$ th stratum. In practice, $r_{h}$ is usually not integer and has to be rounded. In accordance with most of the current literature on the topic, let us assume that the followed-up $r_{h}\left(\subset n_{h(2)}\right)$ units respond on the second call. Moreover, let $U h$ denote a dummy variable which takes value $u h i$ on the $i$ th population unit of stratum $h$ and has mean $\bar{U}_{h}$. Hereafter, $\bar{U}_{h}$ may stand for $Y h ; X h$ or for a second auxiliary variable $Z h$. Let;

$$
\bar{u}_{n_{h(1)}}=\frac{\sum_{i=1}^{n_{h(1)}} u_{i(1)}}{n_{h(1)}}, \bar{u}_{r_{h}}=\frac{\sum_{i=1}^{r_{h(1)}} u_{i(2)}}{r_{h}}
$$

and

$$
\bar{u}_{h}^{*}=\frac{n_{h_{(1)}}}{n_{h}} \bar{u}_{(1) n_{h_{1}}}+\frac{n_{h_{(2)}}}{n_{h}} \bar{u}_{(2) r_{h}}
$$

where $\bar{u}_{(1) n_{h(1)}}$ is mean of $n_{h(1)}$ respondents on first call and $\bar{u}_{(2) r_{h}}$ is mean of $r_{h}$ units respond on the second call, and $\bar{u}_{h}^{*}$ denotes the unbiased Hansen-Hurwitz [1] of $\bar{U}_{h}$ for stratum $h$.

A modified Hansen and Hurwitz [1] unbiased estimator for stratified sampling may be given as,

$$
t_{1}=\sum_{h=1}^{L} P_{h} \bar{u}_{h}^{*}
$$

The variance of $t_{1}$ is,

$$
\operatorname{Var}\left(t_{1}\right)=\sum_{h=1}^{L} \lambda_{h} P_{h}^{2} S_{u_{h}}^{2}+\sum_{h=1}^{L} \lambda_{h}^{*} P_{h}^{2} S_{u_{h(2)}}^{2}
$$

where $S_{u h}^{2}=\sum_{i=1}^{N_{h}}\left(u_{h i}-\bar{U}_{h}\right)^{2} /\left(N_{h}-1\right)$, and $S_{u h(2)}^{2}=\sum_{i=1}^{N_{h(2)}}\left(u_{i}-\bar{U}_{h(2)}\right)^{2} /\left(N_{h(2)}-1\right)$ are the mean square error of the entire group and the non-response group of the study variable with $P_{h}=N_{h} / N, \lambda_{h}=\left(\frac{1}{n_{h}}-\frac{1}{N_{h}}\right), \lambda_{h}^{*}=\left(\frac{f_{h}-1}{n_{h}}\right) W_{h(2)}, W_{h(2)}=N_{h(2)} / N_{h}$, and $f_{h}=n_{h(2)} / r_{h}$. The modified form of ratio and product estimators from stratified random sampling under non-response defined by Cochran [2] may be written as:

$$
t_{2}=\bar{y}_{\mathrm{st}}^{*}\left[\frac{\bar{X}}{\overline{\bar{x}}_{\mathrm{st}}^{*}}\right]\left[\frac{\bar{Z}}{\overline{\bar{z}_{\mathrm{st}}^{*}}}\right]
$$

and

$$
t_{3}=\bar{y}_{\mathrm{st}}^{*}\left[\frac{\bar{x}_{\mathrm{st}}^{*}}{\bar{X}}\right]\left[\frac{\bar{z}_{\mathrm{st}}^{*}}{\bar{Z}}\right]
$$

where $\bar{y}_{\mathrm{st}}^{*}$ and $\bar{x}_{\mathrm{st}}^{*}$ are Hansen-Hurwitz [1] estimators modified to the stratified sampling for population means $\bar{X}$ and $\bar{Y}$ respectively.

The MSE of the estimators $t_{2}$ and $t_{3}$ are given respectively as

$$
\begin{gathered}
\operatorname{MSE}\left(t_{2}\right) \approx \bar{Y}^{2} \sum_{h=1}^{L}\left[\lambda_{h}\left(\frac{S_{h y}^{2}}{\bar{Y}^{2}}+\frac{S_{h x}^{2}}{\bar{X}^{2}}+\frac{S_{h z}^{2}}{\bar{Z}^{2}}-2 \frac{S_{h x y}}{\overline{Y X}}-2 \frac{S_{h y z}}{\bar{Y} \bar{C}}+2 \frac{S_{h y z}}{\bar{X} Z}\right)+\right. \\
\left.\lambda_{h}^{*}\left(\frac{S_{h y(2)}^{2}}{\bar{Y}^{2}}+\frac{S_{h x(2)}^{2}}{\bar{X}^{2}}+\frac{S_{h z(2)}^{2}}{\bar{Z}^{2}}-2 \frac{S_{h x y(2)}}{\overline{Y X}}-2 \frac{S_{h y z(2)}}{\bar{Y} \bar{Z}}+2 \frac{S_{h x z(2)}}{\bar{X} \bar{Z}}\right)\right],
\end{gathered}
$$

and

$$
\operatorname{MSE}\left(t_{3}\right) \approx \bar{Y}^{2} \sum_{h=1}^{L} \lambda_{h}\left(\frac{S_{h y}^{2}}{\bar{Y}^{2}}+\frac{S_{h x}^{2}}{\bar{X}^{2}}+\frac{S_{h z}^{2}}{\bar{Z}^{2}}+2 \frac{S_{h x y}}{\overline{Y X}}+2 \frac{S_{h y z}}{\bar{Y} \bar{Z}}+2 \frac{S_{h y z}}{\bar{X} \bar{Z}}\right)+
$$




$$
\left.\lambda_{h}^{*}\left(\frac{S_{h y(2)}^{2}}{\bar{Y}^{2}}+\frac{S_{h x(2)}^{2}}{\bar{X}^{2}}+\frac{S_{h z(2)}^{2}}{\bar{Z}^{2}}+2 \frac{S_{h x y(2)}}{\overline{Y X}}+2 \frac{S_{h y z(2)}}{\overline{Y Z}}+2 \frac{S_{h x z(2)}}{\overline{X Z}}\right)\right]
$$

Chaudhary et al. [3] presented ratio estimator for stratified sampling when non-response is present only on study variable as,

$$
t_{4}=\bar{y}_{\mathrm{st}}^{*}\left[\frac{a \bar{X}+b}{\alpha\left(a \bar{x}_{\mathrm{st}}+b\right)+(1-\alpha)(a \bar{X}+b)}\right]^{g} \text {, }
$$

The MSE of $t_{3}$ is

$$
\operatorname{MSE}\left(t_{4}\right) \approx \sum_{h=1}^{L} P_{h}^{2} \lambda_{h}\left[S_{y h}^{2}+\alpha^{2} v^{2} g^{2} R^{2} S_{x h}^{2}-2 \alpha v g R \rho_{x y h} S_{x h} S_{y h}\right]+\sum_{h=1}^{L} P_{h}^{2} \lambda_{h}^{*} S_{y h(2)}^{2},
$$

where

$$
v=\frac{a \bar{X}}{a \bar{X}+b}, R=\frac{\bar{Y}}{\bar{X}}
$$

The $\operatorname{MSE}\left(t_{5}\right)$ is minimum for

$$
\alpha=\frac{\sum_{h=1}^{L} P_{h}^{2} \lambda_{h} \rho_{x y h} S_{y h} S_{x h}}{\operatorname{vgR} \sum_{h=1}^{L} P_{h}^{2} \lambda_{h} S_{x h}} .
$$

The aim of this paper is to propose a more generalized class of estimators for estimating population mean considering the non-response in stratified random sampling using two auxiliary variables. Also the purpose is to introduce another improved form of proposed generalised estimator. Another purpose is to determine the optimum size of the sample and the sub-sampling fractions of the non-respondent group for the fixed cost.

\section{PROPOSED GENERALIZED CLASS OF ESTIMATORS}

In this section, following Koyuncu and Kadilar [12] we proposed a generalized class of estimators for estimating a finite population mean in stratified random sampling considering the presence of non-response using two auxiliary variables as,

$$
t_{a}=\bar{y}_{\mathrm{st}}^{*}\left[\frac{a_{X} \bar{X}+b_{X}}{\alpha_{X}\left(a_{X} \bar{x}_{\mathrm{st}}^{*}+b_{X}\right)+\left(1-\alpha_{X}\right)\left(a_{X} \bar{X}+b_{X}\right)}\right]^{g_{X}}\left[\frac{a_{Z} \bar{Z}+b_{Z}}{\alpha_{Z}\left(a_{Z} \bar{z}_{\mathrm{st}}^{*}+b_{X}\right)+\left(1-\alpha_{Z}\right)\left(a_{Z} \bar{Z}+b_{Z}\right)}\right]^{g_{Z}},
$$

where the constants $a_{x} \& a_{z}(\neq 0)$, and $b_{x} \& b_{z}$ are either real numbers or the functions of the auxiliary variable, in form of coefficient of variations, standard deviations, correlation coefficients, skewness or kurtosis from the population, whereas $g_{x}$ and $g_{z}$ are known constants

\begin{tabular}{|c|c|c|c|c|c|c|c|}
\hline $\begin{array}{l}\text { Family of ratio-cum-ratio } \\
\qquad g_{x}=g_{z}=1\end{array}$ & $\begin{array}{c}\text { Family of } \\
\text { product-cum-product } \\
g_{x}=g_{z}=-1\end{array}$ & $a_{X}$ & $\boldsymbol{b}_{X}$ & $a_{Z}$ & $b_{Z}$ & $\alpha_{X}$ & $\alpha_{Z}$ \\
\hline$t_{a}^{1}$ & $t_{a}^{2}$ & 1 & 0 & 1 & 0 & 1 & 1 \\
\hline$t_{a}^{3}$ & $t_{a}^{4}$ & 1 & $\rho_{x y}$ & 1 & $\rho_{y z}$ & 1 & 1 \\
\hline$t_{a}^{5}$ & $t_{a}^{6}$ & $\sigma_{x}$ & 1 & $\sigma_{z}$ & 1 & 1 & 1 \\
\hline$t_{a}^{7}$ & $t_{a}^{8}$ & $\rho_{x y}$ & 1 & $\rho_{y z}$ & 1 & 1 & 1 \\
\hline
\end{tabular}
take the value $(0,1,-1)$ to produce respectively unbiased estimator, different families of ratio-cum-ratio and families of product-cumproduct type estimators, and $\alpha_{x}$ and $\alpha_{z}$ are the constants to be determined such that MSE of the proposed estimator $t_{a}$ is minimum. Taking different values of the constants we may obtain many families of ratio-cum-ratio and families of product-cum-product estimators such as some examples are presented in Table 1.

Table 1 Families of the estimators $t_{a}$. 


\subsection{Bias and MSE of Proposed Generalized Class of Estimator}

To obtain the bias and MSE, we consider

$$
\begin{gathered}
\bar{y}_{s t}^{*}=\sum_{h=1}^{L} P_{h} \bar{y}_{h}^{*}=\bar{Y}\left(1+e_{o}^{*}\right), \bar{x}_{s t}^{*}=\bar{X}\left(1+e_{1}^{*}\right), \bar{z}_{s t}^{*}=\bar{Z}\left(1+e_{2}^{*}\right) \\
E\left(e_{i}^{*}\right)=0 \quad \text { for } \quad i=0,1,2
\end{gathered}
$$

and

$$
\left.\begin{array}{c}
V_{r, s, t}^{*}=\sum_{h=1}^{L} P_{h}^{r+s+t} \frac{E\left(\left(\bar{x}_{h}^{*}-\bar{X}_{h}\right)^{r}\left(\bar{y}_{h}^{*}-\bar{Y}_{h}\right)^{s}\left(\bar{z}_{h}^{*}-\bar{Z}_{h}\right)^{t}\right)}{\bar{X}^{r} \bar{Y}^{s} \bar{Z}^{t}} \\
E\left(e_{o}^{*}\right)^{2}=\frac{1}{\bar{Y}^{2}} \sum_{h=1}^{L} P_{h}^{2}\left(\lambda_{h} S_{y h}^{2}+\lambda_{h}^{*} S_{y h 2}^{2}\right)=V_{020}^{*} \quad E\left(e_{1}^{*}\right)^{2}=\frac{1}{\bar{X}^{2}} \sum_{h=1}^{L} P_{h}^{2}\left(\lambda_{h} S_{x h}^{2}+\lambda_{h}^{*} S_{x h 2}^{2}\right)=V_{200}^{*} \\
E\left(e_{2}^{*}\right)^{2}=\frac{1}{\bar{Z}^{2}} \sum_{h=1}^{L} P_{h}^{2}\left(\lambda_{h} S_{z h}^{2}+\lambda_{h}^{*} S_{z h 2}^{2}\right)=V_{002}^{*} \quad E\left(e_{o}^{*} e_{2}^{*}\right)=\frac{1}{\overline{Y Z}} \sum_{h=1}^{L} P_{h}^{2}\left(\lambda_{h} S_{y z h}+\lambda_{h}^{*} S_{y z h 2}\right)=V_{011}^{*} \\
E\left(e_{o}^{*} e_{1}^{*}\right)=\frac{1}{\overline{Y X}} \sum_{h=1}^{L} P_{h}^{2}\left(\lambda_{h} S_{x y h}+\lambda_{h}^{*} S_{x y h 2}\right)=V_{110}^{*} \quad E\left(e_{1}^{*} e_{2}^{*}\right)=\frac{1}{Z X} \sum_{h=1}^{L} P_{h}^{2}\left(\lambda_{h} S_{x z h}+\lambda_{h}^{*} S_{x z h 2}\right)=V_{101}^{*}
\end{array}\right\}
$$

On rewriting we may get Eq. (11) as

$$
t_{a}=\bar{Y}\left(1+e_{0}^{*}\right)\left[1+\alpha_{x} \nu_{x} e_{1}^{*}\right]^{-g_{x}}\left[1+\alpha_{z} \nu_{z} e_{2}^{*}\right]^{-g_{z}},
$$

where

$$
v_{x}=\frac{a_{x} \bar{X}}{a_{x} \bar{X}+b_{x}} \text { and } v_{z}=\frac{a_{z} \bar{Z}}{a_{z} \bar{Z}+b_{z}} .
$$

We assume that $\left|\alpha_{x} \nu_{x} e_{1}^{*}\right|<1$ and $\left|\alpha_{z} \nu_{z} e_{2}^{*}\right|<1$, so that we may expand the series, $\left[1+\alpha_{x} \nu_{x} e_{1}^{*}\right]^{-g_{x}}$ and $\left[1+\alpha_{z} \nu_{z} e_{2}^{*}\right]^{-g_{z}}$, we get

$$
\begin{gathered}
t_{a}=\bar{Y}\left(1+e_{0}^{*}\right)\left[1-\left(g_{x} \alpha_{x} \nu_{x}\right) e_{1}^{*}+\frac{g_{x}\left(g_{x}+1\right)}{2}\left(\alpha_{x} \nu_{x}\right)^{2} e_{1}^{* 2}+\ldots\right] \\
{\left[1-g_{z} \alpha_{z} v_{z} e_{2}^{*}+\frac{g_{z}\left(g_{z}+1\right)}{2}\left(\alpha_{z} v_{z}\right)^{2} e_{2}^{* 2}+\ldots\right] .}
\end{gathered}
$$

It is assumed that the contribution of terms involving powers in $e_{0}^{*}, e_{1}^{*}$ and $e_{2}^{*}$ higher than two is negligible. We have,

$$
\begin{aligned}
t_{a}-\bar{Y}= & \bar{Y}\left[e_{0}^{*}-\left(g_{x} \alpha_{x} v_{x}\right) e_{1}^{*}-\left(g_{x} \alpha_{x} \nu_{x}\right) e_{0}^{*} e_{1}^{*}+\frac{g_{x}\left(g_{x}+1\right)}{2}\left(\alpha_{x} \nu_{x}\right) e_{1}^{* 2}-\left(g_{z} \alpha_{z} v_{z}\right) e_{2}^{*}\right. \\
& \left.-\left(g_{z} \alpha_{z} v_{z}\right) e_{0}^{*} e_{2}^{*}+\left(g_{x} \alpha_{x} v_{x}\right)\left(g_{z} \alpha_{z} v_{z}\right) e_{1}^{*} e_{2}^{*}+\frac{g_{z}\left(g_{z}+1\right)}{2}\left(\alpha_{z} v_{z}\right)^{2} e_{2}^{* 2}\right] .
\end{aligned}
$$

Taking expectation of Eq. (15), we will get the bias as,

$$
\begin{aligned}
\operatorname{Bias}\left(t_{a}\right)= & {\left[g_{x} \alpha_{x} \nu_{x} V_{110}^{*}+\frac{g_{x}\left(g_{x}+1\right)}{2} \alpha_{x}^{2} v_{x}^{2} V_{200}^{*}\right.} \\
& \left.-\left(g_{z} \alpha_{z} \nu_{z}\right) V_{011}^{*}+\left(g_{x} \alpha_{x} \nu_{x}\right)\left(g_{z} \alpha_{z} \nu_{z}\right) V_{101}^{*}+\frac{g_{z}\left(g_{z}+1\right)}{2}\left(\alpha_{z}^{2} \nu_{z}^{2}\right) V_{002}^{* 2}\right] .
\end{aligned}
$$

we take square of Eq. (15) and retain terms up to the order $n-{ }^{1}$ then we take expectation to get the MSE of the estimator $t a$ as,

$$
\begin{aligned}
\operatorname{MSE}\left(t_{a}\right)=\bar{Y}^{2} & {\left[V_{020}^{*}+\left(g_{x} \alpha_{x} \nu_{x}\right)^{2} V_{200}^{*}+\left(g_{z} \alpha_{z} \nu_{z}\right)^{2} V_{002}^{*}-2\left(g_{x} \alpha_{x} \nu_{x}\right) V_{110}^{*}\right.} \\
& \left.-2\left(g_{z} \alpha_{z} \nu_{z}\right) V_{011}^{*}+2\left(g_{x} \alpha_{x} \nu_{x}\right)\left(g_{z} \alpha_{z} \nu_{z}\right) V_{101}^{*}\right]
\end{aligned}
$$


Minimization of Eq. (17) with respect to $\alpha_{x}$ and $\alpha_{z}$ yields the optimum values as

$$
\alpha_{x}=\frac{\left(V_{011}^{*} V_{101}^{*}-V_{110}^{*} V_{002}\right)}{g_{x} v_{x}\left(V_{101}^{* 2}-V_{002}^{*} V_{200}^{*}\right)} \text { and } \alpha_{z}=\frac{\left(V_{101}^{*} V_{110}^{*}-V_{011}^{*} V_{200}\right)}{g_{z} V_{z}\left(V_{101}^{* 2}-V_{002}^{*} V_{200}^{*}\right)}
$$

Using the Eq. (18), the expression of minimum MSE may be obtained as

$$
\operatorname{MSE}\left(t_{a}\right)=\bar{Y}^{2}\left[V_{020}^{*}-\left(\frac{V_{002}^{*} V_{110}^{* 2}+V_{200}^{*} V_{011}^{* 2}-2 V_{101}^{*} V_{011}^{*} V_{110}^{*}}{V_{200}^{*} V_{002}^{*}-V_{101}^{* 2}}\right)\right] .
$$

For generalized family of ratio-cum-ratio estimators presented in Table 1, we can give the MSE expression in Eq. (17) as,

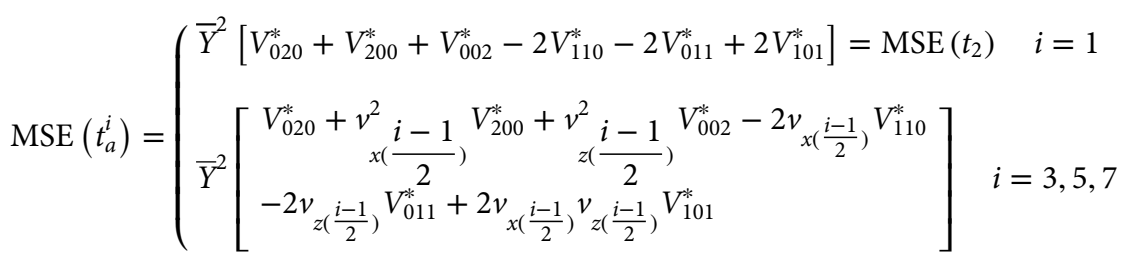

and for generalized family of product-cum-product estimators, the MSE expression can be given as,

$$
\operatorname{MSE}\left(t_{a}^{j}\right)=\left(\begin{array}{l}
\bar{Y}^{2}\left[V_{020}^{*}+V_{200}^{*}+V_{002}^{*}+2 V_{110}^{*}+2 V_{011}^{*}+2 V_{101}^{*}\right]=\operatorname{MSE}\left(t_{3}\right) \quad j=2 \\
\bar{Y}^{2}\left[\begin{array}{c}
V_{020}^{*}+v^{2}{ }_{x\left(\frac{j}{2}-1\right)} V_{200}^{*}+v^{2}{ }_{z\left(\frac{j}{2}-1\right)}^{j} V_{002}^{*}+2 v_{x\left(\frac{j}{2}-1\right)} V_{110}^{*} \\
+2 v_{z\left(\frac{j}{2}-1\right)} V_{011}^{*}+2 v_{x\left(\frac{j}{2}-1\right)} v_{z\left(\frac{j}{2}-1\right)} V_{101}^{*}
\end{array}\right] j=4,6,8
\end{array}\right.
$$

Where

$$
\begin{array}{rlrl}
v_{x 1} & =\frac{\bar{X}}{\bar{X}+\rho_{x y}} & \text { and } & v_{z 1}=\frac{\bar{Z}}{\bar{Z}+\rho_{y z}} \\
v_{x 2}=\frac{\sigma_{x} \bar{X}}{\sigma_{x} \bar{X}+1} & \text { and } & v_{z 2}=\frac{\sigma_{z} \bar{Z}}{\sigma_{z} \bar{Z}+1} \\
v_{x 3}=\frac{\rho_{x y} \bar{X}}{\rho_{x y} \bar{X}+1} & \text { and } & v_{z 3}=\frac{\rho_{y z} \bar{Z}}{\rho_{y z} \bar{Z}+1}
\end{array}
$$

One can think many more estimators from Eq. (11), and the bias and MSE expression for these estimators can be expressed by Eqs. (16) and (17) respectively.

\section{ANOTHER PROPOSED GENERALIZED ESTIMATORS}

In this section, we have shown another improved and generalized form for the estimator $t a$ proposed in Section 3. The proposed estimator $t s$ for estimating population mean is given as,

$$
t_{s}=\eta \bar{y}_{\mathrm{st}}^{*}\left[\frac{a_{X} \bar{X}+b_{X}}{\alpha_{X}\left(a_{X} \bar{x}_{\mathrm{st}}^{*}+b_{X}\right)+\left(1-\alpha_{X}\right)\left(a_{X} \bar{X}+b_{X}\right)}\right]^{g_{X}}\left[\frac{a_{Z} \bar{Z}+b_{Z}}{\alpha_{Z}\left(a_{Z} \bar{z}_{\mathrm{st}}^{*}+b_{X}\right)+\left(1-\alpha_{Z}\right)\left(a_{Z} \bar{Z}+b_{Z}\right)}\right]^{g_{Z}}
$$

or may also be consider as,

$$
t_{s}=\eta \bar{Y}\left(1+e_{0}^{*}\right)\left[1+\alpha_{x} \nu_{x} e_{1}^{*}\right]^{-g_{x}}\left[1+\alpha_{z} v_{z} e_{2}^{*}\right]^{-g_{z}},
$$

We assume that $\left|\alpha_{x} \nu_{x} e_{1}^{*}\right|<1$ and $\left|\alpha_{z} \nu_{z} e_{2}^{*}\right|<1$, so that we may expand the series, $\left[1+\alpha_{x} \nu_{x} e_{1}^{*}\right]^{-g_{x}}$ and $\left[1+\alpha_{z} \nu_{z} e_{2}^{*}\right]^{-g_{z}}$, we get

$$
\begin{gathered}
t_{s}=\eta \bar{Y}\left(1+e_{0}^{*}\right)\left[1-\left(g_{x} \alpha_{x} \nu_{x}\right) e_{1}^{*}+\frac{g_{x}\left(g_{x}+1\right)}{2}\left(\alpha_{x} \nu_{x}\right)^{2} e_{1}^{* 2}+\ldots\right] \\
{\left[1-g_{z} \alpha_{z} \nu_{z} e_{2}^{*}+\frac{g_{z}\left(g_{z}+1\right)}{2}\left(\alpha_{z} \nu_{z}\right)^{2} e_{2}^{* 2}+\ldots\right] .}
\end{gathered}
$$


It is assumed that the contribution of terms involving powers in $e_{0}^{*}, e_{1}^{*}$ and $e_{2}^{*}$ higher than two is negligible. We have,

$$
\begin{gathered}
t_{s}-\bar{Y}=\bar{Y}(\eta-1)+\bar{Y}\left[e_{0}^{*}-\left(g_{x} \alpha_{x} \nu_{x}\right) e_{1}^{*}-\left(g_{x} \alpha_{x} v_{x}\right) e_{0}^{*} e_{1}^{*}+\frac{g_{x}\left(g_{x}+1\right)}{2}\left(\alpha_{x} v_{x}\right) e_{1}^{* 2}-\left(g_{z} \alpha_{z} v_{z}\right) e_{2}^{*}\right. \\
\left.-\left(g_{z} \alpha_{z} v_{z}\right) e_{0}^{*} e_{2}^{*}+\left(g_{x} \alpha_{x} \nu_{x}\right)\left(g_{z} \alpha_{z} v_{z}\right) e_{1}^{*} e_{2}^{*}+\frac{g_{z}\left(g_{z}+1\right)}{2}\left(\alpha_{z} v_{z}\right)^{2} e_{2}^{* 2}\right] .
\end{gathered}
$$

The bias and MSE expressions of $t$ s may be given respectively as,

$$
\operatorname{Bias}\left(t_{s}\right)=\bar{Y}(\eta-1)+\operatorname{Bias}\left(t_{a}\right)
$$

and

$$
\operatorname{MSE}\left(t_{s}\right)=\bar{Y}^{2}(\eta-1)^{2}+\eta^{2} \operatorname{MSE}\left(t_{a}\right)+2 \bar{Y} \eta(\eta-1) \operatorname{Bias}\left(t_{a}\right)
$$

We differentiate Eq. (25) w.r.t $\tilde{\eta}$, and equate it to zero, we get

$$
\tilde{\eta}=\frac{\bar{Y}\left(\bar{Y}+\operatorname{Bias}\left(t_{a}\right)\right)}{\left[\bar{Y}^{2}+\operatorname{MSE}\left(t_{a}\right)+2 \bar{Y} \operatorname{Bias}\left(t_{a}\right)\right]},
$$

On substitution the optimum value of $\eta$ as given in Eq. (26), in the result Eq. (25), the min MSE of the proposed estimator $t s$ is obtained as,

$$
\min \operatorname{MSE}\left(t_{s}\right)=\bar{Y}^{2}-\frac{\bar{Y}^{2}\left(\bar{Y}+\operatorname{Bias}\left(t_{a}\right)\right)^{2}}{\left[\bar{Y}^{2}+\operatorname{MSE}\left(t_{a}\right)+2 \bar{Y} \operatorname{Bias}\left(t_{a}\right)\right]}
$$

Or

$$
\min \operatorname{MSE}\left(t_{s}\right)=\bar{Y}^{2}\left[1-\frac{\left(1+\frac{\operatorname{Bias}\left(t_{a}\right)}{\bar{Y}}\right)^{2}}{\left[1+\frac{\operatorname{MSE}\left(t_{a}\right)}{\bar{Y}^{2}}+2 \frac{\operatorname{Bias}\left(t_{a}\right)}{\bar{Y}}\right]}\right]
$$

\begin{tabular}{|c|c|c|c|c|c|}
\hline $\begin{array}{c}\begin{array}{c}\text { Family of } \\
\text { ratio-cum-ratio }\end{array} \\
\mathrm{g}_{x}=\mathrm{g}_{z}=1 \\
\end{array}$ & $\begin{array}{c}\text { Family of } \\
\text { product-cum-product } \\
\mathrm{g}_{x}=\mathrm{g}_{z}=-1\end{array}$ & $\left(\mathrm{a}_{x}, \mathbf{b}_{x}, \mathbf{a}_{z}, \mathbf{b}_{z}\right)$ & $\alpha_{x}$ & $\alpha_{z}$ & $\eta$ \\
\hline$t_{s}^{1}$ & $t_{s}^{2}$ & $(1,0,1,0)$ & 1 & 1 & $\eta$ \\
\hline$t_{s}^{3}$ & $\begin{array}{l}s \\
t_{s}^{4}\end{array}$ & $\left(1, \rho_{x y}, 1, \rho_{y z}\right)$ & 1 & 1 & $\eta$ \\
\hline$t_{s}^{5}$ & $t_{s}^{6}$ & $\left(\sigma_{x}, 1,1, \sigma_{y}\right)$ & 1 & 1 & $\eta$ \\
\hline$t_{s}^{7}$ & $t_{s}^{8}$ & $\left(\rho_{x y}, 1,1, \rho_{y z}\right)$ & 1 & 1 & $\eta$ \\
\hline
\end{tabular}

We can think many more improved estimators taking different values of the constants in Eq. (20), such as some example are given in Table 2. The MSE of the ratio-cum-ratio and product-cum-product estimators given in Table 2 can be given using Eq. (27) as,

$$
\begin{aligned}
& \operatorname{MSE}\left(t_{s}^{i}\right)=\bar{Y}^{2}\left[1-\frac{\left(1+\frac{\operatorname{Bias}\left(t_{a}^{i}\right)}{\bar{Y}}\right)^{2}}{\left[1+\frac{\operatorname{MSE}\left(t_{a}^{i}\right)}{\bar{Y}^{2}}+2 \frac{\operatorname{Bias}\left(t_{a}^{i}\right)}{\bar{Y}}\right]}\right] \text { for } i=1,3,5,7 \\
& \operatorname{MSE}\left(t_{s}^{j}\right)=\bar{Y}^{2}\left[1-\frac{\left(1+\frac{\operatorname{Bias}\left(t_{a}^{j}\right)}{\bar{Y}}\right)^{2}}{\left[1+\frac{M S E\left(t_{a}^{j}\right)}{\bar{Y}^{2}}+2 \frac{\operatorname{Bias}\left(t_{a}^{j}\right)}{\bar{Y}}\right]}\right] \text { for } j=2,4,6,8
\end{aligned}
$$

Table 2 Families of the proposed estimator $t$. 


\section{MATHEMATICAL COMPARISON}

In this section, the MSE of the suggested family of estimator has been compared with the mean estimator, stratified ratio estimator and the class of estimators. Let us consider following notations as:

$$
\begin{aligned}
& A_{i}=\left[\begin{array}{c}
v^{2}{ }_{i-1} V_{200}^{*}+v^{2}{ }_{z\left(\frac{i-1}{2}\right)} V_{002}^{*}-2 v_{x\left(\frac{i-1}{2}\right)} V_{110}^{*} \\
-2 v_{z\left(\frac{i-1}{2}\right)} V_{011}^{*}+2 v_{x\left(\frac{i-1}{2}\right)} v_{z\left(\frac{i-1}{2}\right)} V_{101}^{*}
\end{array}\right] \quad i=3,5,7 \\
& A_{j}=\left[\begin{array}{ccc}
v^{2}{ }_{j} & V_{200}^{*}+v^{2}{ }_{j} & V_{002}^{*}+2 v_{x\left(\frac{j}{2}-1\right)} V_{110}^{*} \\
x\left(\frac{2}{2}-1\right) & z\left(\frac{2}{2}-1\right) & \\
+2 v_{z\left(\frac{j}{2}-1\right)} V_{011}^{*}+2 v_{x\left(\frac{j}{2}-1\right)} v_{z\left(\frac{j}{2}-1\right)} V_{101}^{*}
\end{array}\right] \quad j=4,6,8 \\
& B_{1}=v_{z\left(\frac{i-1}{2}\right)}^{2} V_{002}^{*}-2 v_{z\left(\frac{i-1}{2}\right)} V_{011}^{*}-V_{200}^{*}-V_{002}^{*}+2 V_{110}^{*}+2 V_{011}^{*}-2 V_{101}^{*} \\
& B_{2}=\left(\frac{V_{002}^{*} V_{110}^{* 2}+V_{200}^{*} V_{011}^{* 2}-2 V_{101}^{*} V_{011}^{*} V_{110}^{*}}{V_{200}^{*} V_{002}^{*}-V_{101}^{* 2}}\right)
\end{aligned}
$$

and

$$
C^{i}=\frac{\left(1+\frac{\operatorname{Bias}\left(t_{a}^{i}\right)}{\bar{Y}}\right)^{2}}{\left[1+\frac{\operatorname{MSE}\left(t_{a}^{i}\right)}{\bar{Y}^{2}}+2 \frac{\operatorname{Bias}\left(t_{a}^{i}\right)}{\bar{Y}}\right]}
$$

The efficiency conditions may be written as:

i.

$$
\begin{array}{ll}
\operatorname{Var}\left(t_{1}\right)>\operatorname{MSE}\left(t_{a}^{i}\right) & i=3,5,7 \\
\text { If } & \\
V_{020}^{*}-A_{i}>0 &
\end{array}
$$

ii.

$$
\begin{aligned}
& \operatorname{MSE}\left(t_{2}\right)>\operatorname{MSE}\left(t_{a}^{i}\right) \\
& \text { If } \\
& \min \left(\frac{-A_{i} \pm \sqrt{A_{i}^{2}-B_{1} V_{200}^{*}}}{V_{200}^{*}}\right) \leq v_{x\left(\frac{i-1}{2}\right)} \leq \max \left(\frac{-A_{i} \pm \sqrt{A_{i}^{2}-B_{1} V_{200}^{*}}}{V_{200}^{*}}\right) \\
& {[14 p t]}
\end{aligned}
$$

iii.

$$
\begin{aligned}
& \operatorname{Var}\left(t_{1}\right)>\min \operatorname{MSE}\left(t_{a}^{i}\right) \\
& \text { If } \\
& B_{2}>0
\end{aligned}
$$

iv.

$$
\begin{aligned}
& \operatorname{MSE}\left(t_{2}\right)>\min \operatorname{MSE}\left(t_{a}^{i}\right) \\
& \text { If } \\
& B_{2}>V_{200}^{*}+V_{002}^{*}-2 V_{110}^{*}-2 V_{011}^{*}+2 V_{101}^{*}
\end{aligned}
$$

v.

$$
\begin{aligned}
& \operatorname{Var}\left(t_{1}\right)>\operatorname{MSE}\left(t_{s}^{i}\right) \\
& \text { If } \\
& C^{i}>1-V_{020}^{*}
\end{aligned}
$$


vi.

$$
\begin{aligned}
& \operatorname{MSE}\left(t_{2}\right)>\operatorname{MSE}\left(t_{s}^{i}\right) \\
& \text { If } \\
& C^{i}>1-\left(V_{020}^{*}+V_{200}^{*}+V_{002}^{*}-2 V_{110}^{*}-2 V_{011}^{*}+2 V_{101}^{*}\right)
\end{aligned}
$$

vii.

$$
\begin{aligned}
& \min \operatorname{MSE}\left(t_{a}^{i}\right)>\min \operatorname{MSE}\left(t_{s}^{i}\right) \\
& \text { If } \\
& C^{i}>1+B_{2}-V_{020}^{*}
\end{aligned}
$$

\section{COST FUNCTION AND SAMPLE SIZE ESTIMATION}

Let us assume a linear cost function, the total cost of the sample survey could be written as

$$
C^{\prime}=\sum_{h=1}^{L} c_{h 0} n_{h}+\sum_{h=1}^{L} c_{h 1} n_{h}+\sum_{h=1}^{L} c_{h 2} r_{h}
$$

where $c h_{0}$ denotes the per unit cost of making first attempt, $c h_{1}$ denotes per unit cost for processing the result of all characteristics in first attempt and chh denotes the per unit cost for processing the result of all characteristics in second attempt in the $h$ th stratum.

The total expected cost of the survey could be given as

$$
C=E\left(C^{\prime}\right)=\sum_{h=1}^{L}\left(c_{h 0}+c_{h 1} W_{h 1}\right) n_{h}+\sum_{h=1}^{L} c_{h 2} r_{h}
$$

\subsection{Cost Function for Estimator $t_{a}$}

Let us consider the function for estimator $t a$

$\varphi\left(n_{h}, r_{h}, \delta\right)=\operatorname{Var}\left(t_{a}\right)+\delta\left\{C-C_{0}\right\}$

$$
\begin{aligned}
& \varphi\left(n_{h}, r_{h}, \delta\right)=\bar{Y}^{2}\left[V_{020}^{*}+u_{X}^{2} V_{200}^{*}+u_{Z}^{2} V_{002}^{*}-2 u_{X} V_{110}^{*}-2 u_{Z} V_{011}^{*}+2 u_{X} u_{Z} V_{101}^{*}\right] \\
& +\delta\left[\sum_{h=1}^{L}\left(c_{h 0}+c_{h 1} W_{h 1}\right) n_{h}+\sum_{h=1}^{L} c_{h 2} r_{h}-C_{0}\right]
\end{aligned}
$$

Let

$$
S_{h}=\left(\frac{1}{\bar{Y}^{2}} S_{y_{h}}^{2}+\frac{1}{\bar{X}^{2}} S_{x_{h}}^{2}+\frac{1}{\bar{Z}^{2}} S_{z_{h}}^{2}-2 u_{x} \frac{1}{\overline{X Y}} S_{x y_{h}}-2 u_{z} \frac{1}{\bar{Y} \bar{Z}} S_{y z_{h}}+2 u_{x} u_{z} \frac{1}{\bar{X} \bar{Z}} S_{x z_{h}}\right)
$$

and

$$
S_{h}^{*}=\left(\frac{1}{\bar{Y}^{2}} S_{y_{h}}^{* 2}+\frac{1}{\bar{X}^{2}} S_{x_{h}}^{* 2}+\frac{1}{\bar{Z}^{2}} S_{z_{h}}^{* 2}-2 u_{x} \frac{1}{\overline{X Y}} S_{x y_{h}}^{*}-2 u_{z} \frac{1}{\bar{Y} \bar{Z}} S_{y z_{h}}^{*}+2 u_{x} u_{z} \frac{1}{\bar{X} \bar{Z}} S_{x z_{h}}^{*}\right)
$$

So we can write Eq. (39) as

$$
\varphi\left(n_{h}, r_{h}, \delta\right)=\bar{Y}^{2} \sum_{h=1}^{L} P_{h}^{2}\left[\left(\frac{1}{n_{h}}-\frac{1}{N_{h}}\right) S_{h}+\left(\frac{W_{2 h}\left(f_{h}-1\right)}{r_{h}}\right) S_{h}^{*}\right]+\delta\left[\sum_{h=1}^{L}\left(c_{h 0}+c_{h 1} W_{h 1}\right) n_{h}+\sum_{h=1}^{L} c_{h 2} r_{h}-C_{0}\right]
$$

Where $\delta$ is lagrangian multiplier. On differentiating Eq. (41) we can get the values of $n h, r h$, and $\delta$ as,

$$
n_{h}=\sqrt{\frac{\bar{Y}^{2} P_{h}^{2} S_{h}}{\delta\left(c_{h 0}+c_{h 1} W_{h 1}\right)}}
$$

Also

$$
r_{h}=\sqrt{\frac{\bar{Y}^{2} P_{h}^{2}\left[W_{2 h}\left(f_{h}-1\right) S_{h}^{*}\right]}{\delta c_{h 2}}}
$$




$$
\frac{C_{0}}{\bar{Y} \sum_{h=1}^{L} P_{h}\left[\sqrt{\left(c_{h 0}+c_{h 1} W_{h 1}\right) S_{h}}+\sqrt{\left[W_{2 h}\left(f_{h}-1\right) S_{h}^{*}\right] c_{h 2}}\right]}=\frac{1}{\sqrt{\delta}}
$$

Let

$$
\begin{gathered}
D=\sum_{h=1}^{L} P_{h}\left[\sqrt{\left(c_{h 0}+c_{h 1} W_{h 1}\right) S_{h}}+\sqrt{\left[W_{2 h}\left(f_{h}-1\right) S_{h}^{*}\right] c_{h 2}}\right] \\
\frac{1}{\sqrt{\delta}}=\frac{C_{0}}{\bar{Y} D}
\end{gathered}
$$

Subsituting $\delta$ from Eqs. (44) to (42), we have

$$
n_{h}^{\prime}=\frac{C_{0} \sqrt{P_{h}^{2} S_{h}}}{D \sqrt{\left(c_{h 0}+c_{h 1} W_{h 1}\right)}}
$$

Similarly, substitute the value of $\delta$ from Eqs. (44) to (43) we have

$$
r_{h}^{\prime}=\frac{C_{0} \sqrt{P_{h}^{2}\left[W_{2 h}\left(f_{h}-1\right) S_{h}^{*}\right]}}{D \sqrt{c_{h 2}}}
$$

\section{EMPIRICAL STUDY}

In order to see the performance of the suggested family of estimators as compare to class of estimators under stratified random sampling. The statistics of the two stratified populations have been given in Table 3.

Population-I: [Source: Koyuncu and Kadilar [9]]

We consider No. of teachers as study variable (Y), No. of students as auxiliary variable (X), and No. of classes in primary and secondary schools as another auxiliary variable $(Z)$ for 923 districts at six 6 regions (1: Marmara, 2: Agean, 3: Mediterranean, 4: Central Anatolia, 5: Black Sea, and 6: East and Southeast Anatolia) in Turkey in 2007.

Population-II: [source: detailed livelihood assessment of flood affected districts of Pakistan September 2011, Food Security Cluster, Pakistan]

We consider food expenditure as study variable $(Y)$, household earn as auxiliary variable $(X)$, and total expenditure in May $(2011)$ as another auxiliary variable $(Z)$ for (6 940) male and (1 678) female households in flood affected districts of Pakistan.

Neyman allocation has been used in order to allocate sample sizes to different strata in the two populations separately as:

$$
n_{h}=n \frac{N_{h} S_{h}}{\sum_{h=1}^{L} N_{h} S_{h}}
$$

From Table 3, we observe a positive correlation among study variable and the auxiliary variable in order to use ratio estimators for estimating population mean. The comparison of the proposed estimators have been made with respect to Hansen-Hurwtiz [1] and ratio estimators modified for stratified sampling. The information for the two stratified populations is given in Table 3.

The MSE values of the proposed class of estimators are computed in Table 4 two different data sets. The percent relative efficiencies of the estimators are given in Table 5. Efficiency of each estimator has been tested by increasing the non-response rate from $10 \%$ to $30 \%$ each with three different values of $f h(2,2.5 \& 3)$. From Table 4 it is observed that MSE's of the estimators are increased if non-response increases from $10 \%$ to $30 \%$ however from Table 5 it is noticed that PRE of the proposed estimator is also increased if non-response increases from $10 \%$ to $30 \%$ which shows that the proposed estimators $t a$, and $t s$ as compare to the existing estimators $\left(t_{1}\right.$ and $\left.t_{2}\right)$ can perform more efficiently even at higher non-response rate. If we compare proposed estimators $t a$ and $t s$ with each other, we observe that MSE values of the proposed estimators $t s$ are smaller than the MSE values of proposed estimator $t a$ at each non-response rate. If we compare the two proposed estimators $t a$ and $t s$ on basis their PRE values, the conclusion can be drawn that $t s$ is an improved form of $t a$. 
Table 3 Data statistics for the two populations.

\begin{tabular}{|c|c|c|c|c|c|c|c|c|c|c|}
\hline \multirow{2}{*}{\multicolumn{2}{|c|}{ Stratum $(h)$}} & \multicolumn{6}{|c|}{ Population-I } & \multicolumn{3}{|c|}{ Population-II } \\
\hline & & 1 & 2 & 3 & 4 & 5 & 6 & 1 & 2 & 3 \\
\hline \multirow{12}{*}{ 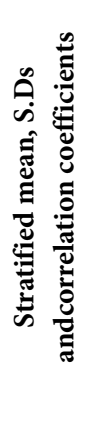 } & $N_{h}$ & 127 & 117 & 103 & 170 & 205 & 201 & 21 & 34 & 26 \\
\hline & $n_{h}$ & 31 & 21 & 29 & 38 & 22 & 39 & 06 & 04 & 02 \\
\hline & $n_{h}^{\prime}$ & 70 & 50 & 75 & 95 & 70 & 90 & 15 & 17 & 08 \\
\hline & $S_{y h}^{n}$ & 883.84 & 644.92 & 1033.40 & 810.58 & 403.65 & 711.72 & 12.14 & 8.34 & 5.47 \\
\hline & Sxh & 30486.7 & 15180.77 & 27549.69 & 18218.93 & 8497.77 & 23094.14 & 76.71 & 31.94 & 49.55 \\
\hline & Szh & 555.58 & 365.46 & 612.95 & 458.03 & 260.85 & 397.05 & 19.48 & 07.10 & 13.21 \\
\hline & $\bar{Y}_{h}$ & 703.74 & 413 & 573.17 & 424.66 & 267.03 & 393.84 & 37.55 & 37.25 & 26.39 \\
\hline & $\bar{X}_{h}$ & 20804.59 & 9211.79 & 14309.30 & 9478.85 & 5569.95 & 12997.59 & 116.57 & 093.00 & 26.39 \\
\hline & $\bar{Z}_{h}^{n}$ & 498.28 & 318.33 & 431.36 & 311.32 & 227.20 & 313.71 & 114.14 & 106.50 & 118.88 \\
\hline & $\rho_{x y h}$ & 0.9360 & 0.9960 & 0.9940 & 0.9830 & 0.9890 & 0.9650 & 0.7914 & 0.8339 & 0.7696 \\
\hline & $\rho_{x z h}$ & 0.9396 & 0.9696 & 0.9770 & 0.9640 & 0.9670 & 0.9960 & 0.9894 & 0.8820 & 0.9669 \\
\hline & $\rho_{y z h}$ & 0.9790 & 0.9760 & 0.9840 & 0.9830 & 0.9640 & 0.9830 & 0.7781 & 0.6651 & 0.5935 \\
\hline \multirow{6}{*}{ 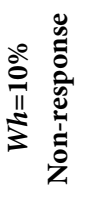 } & $\mathrm{Syh}_{2}$ & 510.57 & 386.77 & 1872.88 & 1603.30 & 264.19 & 497.84 & 08.66 & 10.05 & 03.95 \\
\hline & $\mathrm{Sxh}_{2}$ & 9446.93 & 9198.29 & 52429.99 & 34794.9 & 4972.56 & 12485.10 & 42.14 & 13.28 & 74.22 \\
\hline & $\mathrm{Szh}_{2}$ & 303.92 & 278.51 & 960.71 & 821.29 & 190.85 & 287.99 & 6.25 & 5.20 & 20.53 \\
\hline & $\rho_{x y 2}$ & 0.9961 & 0.9975 & 0.9998 & 0.9741 & 0.9950 & 0.9284 & 0.9997 & 0.9995 & 0.9840 \\
\hline & $\rho_{x z 2}$ & 0.9901 & 0.9895 & 0.9964 & 0.9609 & 0.9865 & 0.9752 & 0.9707 & 1.0000 & 0.9999 \\
\hline & $\rho_{y z 2}$ & 0.9931 & 0.9871 & 0.9972 & 0.9942 & 0.9850 & 0.9647 & 0.9649 & 0.9996 & 0.9819 \\
\hline \multirow{6}{*}{ 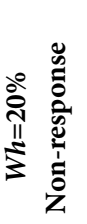 } & $\mathrm{Syh}_{2}$ & 396.77 & 406.15 & 1654.40 & 1333.35 & 335.83 & 903.91 & 7.96 & 8.47 & 4.06 \\
\hline & $\mathrm{Sxh}_{2}$ & 7439.16 & 8880.46 & 45784.78 & 29219.30 & 6540.43 & 28411.44 & 36.50 & 25.82 & 59.32 \\
\hline & $S z h_{2}$ & 244.56 & 274.42 & 965.42 & 680.28 & 214.49 & 469.86 & 5.20 & 8.18 & 16.54 \\
\hline & $\rho_{x y 2}$ & 0.9954 & 0.9931 & 0.9960 & 0.9761 & 0.9966 & 0.9869 & 0.9905 & 0.8026 & 0.8601 \\
\hline & $\rho_{x z 2}$ & 0.9897 & 0.9884 & 0.9789 & 0.9629 & 0.9820 & 0.9825 & 0.9623 & 0.9858 & 0.9956 \\
\hline & $\rho_{y z 2}$ & 0.9898 & 0.9798 & 0.9846 & 0.9940 & 0.9818 & 0.9874 & 0.9297 & 0.8062 & 0.8112 \\
\hline \multirow{6}{*}{ 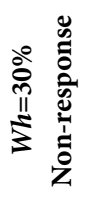 } & $\mathrm{Syh}_{2}$ & 500.26 & 356.95 & 1383.70 & 1193.47 & 289.41 & 825.24 & 12.70 & 09.86 & 4.50 \\
\hline & $\mathrm{Sxh}_{2}$ & 14017.99 & 7812.00 & 38379.77 & 26090.60 & 5611.32 & 24571.95 & 37.69 & 24.02 & 52.26 \\
\hline & $S z h_{2}$ & 284.44 & 247.63 & 811.21 & 631.28 & 188.30 & 437.90 & 9.42 & 6.83 & 14.54 \\
\hline & $\rho_{x y 2}$ & 0.9639 & 0.9919 & 0.9955 & 0.9801 & 0.9961 & 0.9746 & 0.9288 & 0.8335 & 0.8275 \\
\hline & $\rho_{x z 2}$ & 0.9107 & 0.9848 & 0.9771 & 0.9650 & 0.9794 & 0.9642 & 0.9062 & 0.8859 & 0.9907 \\
\hline & $\rho_{y z 2}$ & 0.9739 & 0.9793 & 0.9839 & 0.9904 & 0.9799 & 0.9829 & 0.9696 & 0.5877 & 0.7542 \\
\hline
\end{tabular}

Table 4 MSEs values of estimators.

\begin{tabular}{lccccc}
\hline & & \multicolumn{3}{c}{ MSEs of the different existing estimators and Proposed class of estimators } \\
\hline $\boldsymbol{W h}$ & $\boldsymbol{f h}$ & $\boldsymbol{t}_{\mathbf{1}}$ & $\boldsymbol{t}_{\mathbf{2}}$ & $\boldsymbol{t}_{\boldsymbol{a}}^{\text {min }}$ & $\boldsymbol{t}_{\boldsymbol{s}}^{\text {min }}$ \\
\hline \multirow{3}{*}{$10 \%$} & 2 & 2143.99 & 1411.42 & 76.1190 & $\mathbf{7 6 . 0 8 8 3}$ \\
& 2.5 & 2370.94 & 1501.89 & 81.7661 & $\mathbf{8 1 . 7 3 0 4}$ \\
& 3 & 2597.83 & 1592.34 & 87.1097 & $\mathbf{8 7 . 0 6 9 1}$ \\
$20 \%$ & 2 & 2540.33 & 1667.89 & 83.2651 & $\mathbf{8 3 . 2 2 8 4}$ \\
& 2.5 & 2965.45 & 1886.62 & 92.3106 & $\mathbf{9 2 . 2 6 4 1}$ \\
& 3 & 3390.54 & 2105.42 & 101.171 & $\mathbf{1 0 1 . 1 1 6}$ \\
$30 \%$ & 2 & 2703.09 & 1753.48 & 88.1595 & $\mathbf{8 8 . 1 1 7 9}$ \\
& 2.5 & 3209.59 & 2015.08 & 99.6831 & $\mathbf{9 9 . 6 2 9 1}$ \\
& 3 & 3716.08 & 2276.639 & 110.882 & $\mathbf{1 1 0 . 8 1 5}$ \\
\hline
\end{tabular}

Table 5 PRE values of estimators.

\begin{tabular}{cccccc}
\hline & & \multicolumn{3}{c}{ PREs of the different existing estimators and Proposed class of estimators } \\
\hline $\boldsymbol{W h}$ & $\boldsymbol{f h}$ & $\boldsymbol{t}_{\mathbf{1}}$ & $\boldsymbol{t}_{\mathbf{2}}$ & $\boldsymbol{t}_{\boldsymbol{a}}^{\text {min }}$ & $\boldsymbol{t}_{\boldsymbol{s}}^{\text {min }}$ \\
\hline \multirow{3}{*}{$10 \%$} & 2 & 100 & 151.90305 & 2816.6292 & $\mathbf{2 8 1 7 . 7 6 5 7}$ \\
& 2.5 & 100 & 157.86376 & 2899.6614 & $\mathbf{2 9 0 0 . 9 2 7 9}$ \\
& 3 & 100 & 163.14543 & 2982.2511 & $\mathbf{2 9 8 3 . 6 4 1 7}$ \\
$20 \%$ & 2 & 100 & 152.30801 & 3050.8941 & $\mathbf{3 0 5 2 . 2 3 9 4}$ \\
& 2.5 & 100 & 157.18322 & 3212.4696 & $\mathbf{3 2 1 4 . 0 8 8 7}$ \\
& 3 & 100 & 161.03865 & 3351.2963 & $\mathbf{3 3 5 3 . 1 1 9 2}$ \\
$30 \%$ & 2 & 100 & 154.15574 & 3066.1358 & $\mathbf{3 0 6 7 . 5 8 3 3}$ \\
& 2.5 & 100 & 159.27854 & 3219.7935 & $\mathbf{3 2 2 1 . 5 3 8 7}$ \\
& 3 & 100 & 163.22658 & 3351.3826 & $\mathbf{3 3 5 3 . 4 0 8 8}$ \\
\hline
\end{tabular}




\section{CONCLUSION}

From the results of the numerical study, we infer that both the proposed estimators using auxiliary information perform more efficiently (with substantial gain in precision) than $t_{1}$ and $t_{2}$. Hence proposed estimators are recommended for their practical use.

\section{REFERENCES}

1. M.H. Hansen, W.N. Hurwitz, J. Am. Stat. Assoc. 41(236) (1946), 517-529.

2. W.G. Cochran, Sampling Techniques, Third ed., John Wiley, New York, 1977.

3. M.K. Chaudhary, R. Singh, R.K. Shukla, M. Kumar, F. Smarandache, Pak. J. Stat. Oper. Res. 5(1) (2009), 47-54.

4. A. Haq, J. Shabbir, Commun. Stat. Theory Methodol. 42(1) (2013), 782-799.

5. A. Sanaullah, M. Noor-ul-Amin, M. Hanif, Pak. J. Stat. 31(1) (2015), 71-94.

6. W.G. Cochran, J. Am. Stat. Assoc. 37 (1942), 199-212.

7. C. Kadilar, H. Cingi, Biom. J. 45(2) (2003), 218-225.

8. J. Shabbir, S. Gupta, Am. J. Math. Manag. Sci. 25(3-4) (2005), 293-311.

9. N. Koyuncu, C. Kadilar, J. Stat. Plan. Inference. 139(8) (2009b), 2552-2558.

10. A. Sanaullah, H. Khan, H.A. Ali, R. Singh, J. Reliab. Stat. Stud. 5(2) (2012), 119-132.

11. A. Sanaullah, H.A. Ali, M. Noor ul Amin, M. Hanif, Appl. Math. Comput. 226 (2014), 541-547.

12. N. Koyuncu, C. Kadilar, Commun Stat Theory Methodol. 38(1) (2009a), 2398-2417. 